\title{
E-LABS - LEARNING WITH AUTHENTIC DATA
}

\author{
Marjorie G. Bardeen, Mitchell Wayne \\ ${ }^{1}$ Fermi National Accelerator Laboratory (United States) \\ ${ }^{2}$ University of Notre Dame (United States)
}

\begin{abstract}
e-Labs - Learning with authentic experimental data from cosmic rays and the Compact Muon Solenoid (CMS) at the European Organization for Nuclear Research (CERN) and seismic data from the Laser Interferometer Gravitational-wave Observatory (LIGO)
\end{abstract}

We describe e-Labs, our online laboratories that use the Internet in high school classes. We discuss the success teachers have had providing an opportunity for students to:

- Organize and conduct authentic research.

- Experience the environment of scientific collaborations.

- Possibly make real contributions to a burgeoning scientific field.

We've created projects that are problem-based, student driven and technology dependent. Students reach beyond classroom walls to explore data with other students and experts and share results, publishing original work to a worldwide audience. Students can discover and extend the research of other students, modeling the processes of modern, large-scale research projects.

From start to finish e-Labs are student-led, teacher-guided projects. Students need only a Web browser to access computing techniques employed by professional researchers. A Project Map with milestones allows students to set the research plan rather than follow a step-by-step process common in other online projects. Most importantly, e-Labs build the learning experience around the students' own questions and let them use the very tools that scientists use.

Students contribute to and access shared data, most derived from professional research databases. They use common analysis tools, store their work and use metadata to discover, replicate and confirm the research of others. This is where real scientific collaboration begins. Using online tools, students correspond with other research groups, post comments and questions, prepare summary reports, and in general participate in the part of scientific research that is often left out of classroom experiments.

Teaching tools such as student and teacher logbooks, pre- and post-tests and an assessment rubric aligned with learner outcomes help teachers guide student work. Constraints on interface designs and administrative tools such as registration databases give teachers the "one-stop-shopping" they seek for multiple e-Labs. Teaching and administrative tools also allow us to track usage and assess the impact on student learning.

Keywords: Browser-based labs, problem-based, technology dependent, student led, teacher guided, scientific investigations.

\section{INTRODUCTION}

e-Labs [1] are collaborative online learning environments where high school students use authentic research data in scientific investigations. Their investigations are collaborative, student-driven and technology-dependent. Currently 1344 active teachers are registered for three e-Labs:

1. The CMS e-Lab provides data from over a million proton-proton collisions in the Compact Muon Solenoid (CMS) experiment at the European Organization for Nuclear Research (CERN) [2].

2. Currently, the Cosmic Ray e-Lab provides over 75,000 days of muon data from QuarkNet's [3] network of classroom detectors. Data is added daily.

3. The LIGO e-Lab provides seismic data from the Laser Interferometer Gravitational-wave Observatory (LIGO) [4].

The need for this learning environment developed from an interest of teachers and physicists for students to be able to conduct cosmic ray shower studies. QuarkNet had developed a classroom cosmic ray detector that used a data acquisition card (DAQ) developed by Fermilab engineers and 
technicians. The detector's Global Positioning System (GPS) provided geometry and time stamps to nanoseconds. If students could combine data from a number of sites or from different locations in their schools, they could look for coincident hits on multiple detectors signalling a cosmic ray shower. To support such a study, students needed access to a common dataset and common analysis tools, which led to the design and implementation of what became an e-Lab.

The project began as a case study. Educators and physicists collaborated with computer scientists to design the first e-Lab. They had assistance from an instructional designer. Outside evaluators conducted implementation studies as formative assessment to improve the e-Lab. The composition of the design team was crucial to develop an e-Lab that was user-friendly, made data readily available, supported online analysis tools, and was technically robust and reliable.

The design required a technology infrastructure appropriate for schools and novice users. Educators proposed the following design requirements for teachers:

- Support one-stop shopping.

- Require only a web browser.

- Support guided inquiry.

- Include a hands-on experience.

- Follow learner outcomes.

- $\quad$ Align assessment tools \& strategies with learner outcomes.

- Include professional development \& help desk.

- Build a professional learning community.

- Align with national standards.

Primary design requirements for students included a user-friendly interface and an opportunity to conduct their own investigation without the step-by-step design of most online labs. We also wanted to provide opportunities for students to collaborate with other students-to access, process and publish data, report and share results, to replicate and confirm the work of others and to have online discussions with one another about their work.

CMS physicists took a beautiful existing online analysis tool, iSpy, (Fig. 1) and added features to make it appropriate for "novice" users such as high school students.

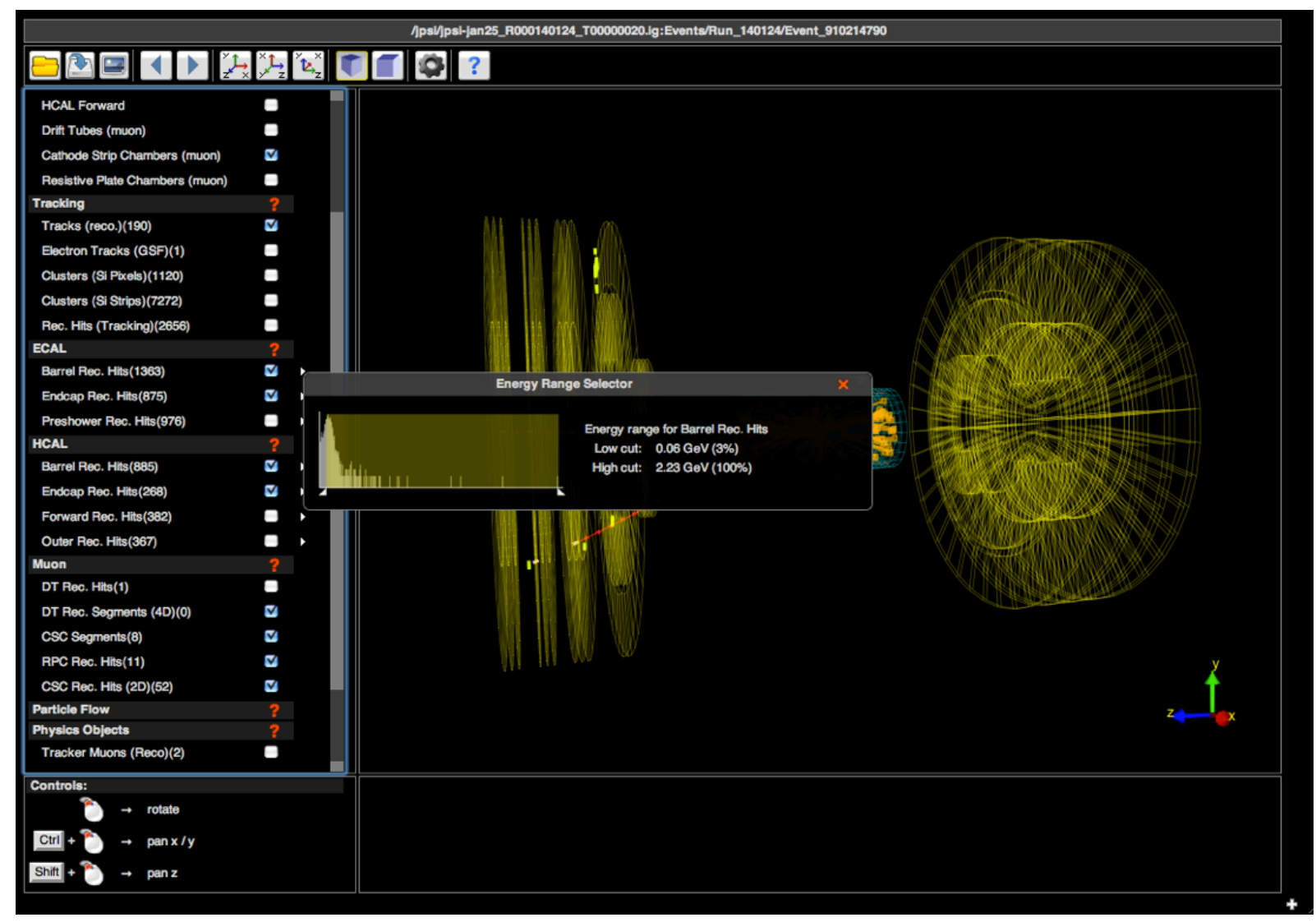

Figure 1. ispy, CMS analysis tool. 
In fact, QuarkNet pioneered the use of data from CERNs Large Hadron Collider (LHC) [5] for high school students and demonstrated the value of making large datasets available for education. QuarkNet developed its own analysis tools for students to collect, upload and analyse cosmic ray data, and LIGO modified existing tools to function online for students. When Advanced LIGO came online in September 2015, the e-Lab tools had to be re-aligned for new datasets.

Computer specialists implemented an overall design, placing a learning environment on top of the then-emerging Grid infrastructure developed by GriPhyN and iVDgL. With skillful design, technology was able to enhance teaching and learning, allowing "apprentice scientists" to manage their own investigations and teachers to facilitate learning, track progress and assess student work. Details on the original design are described in a paper, The QuarkNet/Grid Collaboration Learning e-Lab. [6]

Based on our innovative approach, we received support to modify the code to support multiple e-Labs. This new project was called Interactions in Understanding the Universe (I2U2) [7], supported by the U.S. National Science Foundation (NSF). Once that grant was complete, efforts to maintain and enhance the e-Labs returned to QuarkNet.

\section{BACKGROUND}

e-Labs are aligned with current United States science education standards, The Next Generation Science Standards (NGSS) [9]. Also e-Labs address the interests and needs of high school physics teachers participating in QuarkNet, a long-term professional development program. QuarkNet has incorporated the e-Labs in a searchable online collection of activities organized around data strands that help students develop an understanding about how scientists make discoveries. The Data Portfolio [8] organizes activities by data strand and level of student engagement. Activities differ in complexity and sophistication-tasks in Level 1 are simpler than those in Levels 2 and 3. e-Labs are Level 3 activities. While each level can be explored individually, students who start in one level and progress to more complex levels experience increasingly engaging and challenging tasks. Activities are searchable by strand, level, topic, and NGSS science and engineering practices.

\subsection{QuarkNet}

In its $18^{\text {th }}$ year, QuarkNet is embedded in the particle physics research community. QuarkNet partners high school science teachers and their students with physicists working in experiments at the scientific frontier. These experiments are searching for answers to fundamental questions about the origin of mass, the dimensionality of spacetime and the nature of symmetries that govern physical processes. Physicists and teachers bring the excitement of modern physics — dark matter, dark energy, the Higgs Boson, even gravitational waves-to high school physics classrooms.

QuarkNet is led by a national group of staff teachers, educators and physicists with many years of experience in professional development workshops and institutes, instructional materials development and teacher research programs. The project includes 50 centers at universities and research labs in 26 states and Puerto Rico where 82 mentors and over 550 teachers collaborate locally to enhance the teaching and learning of physics.

The program develops teachers' knowledge and skills to support student investigations that model scientific research. QuarkNet offers research internships for teachers and students, masterclasses [9] for students, research-based workshops and ongoing support for teachers, and provides instructional materials and access to online datasets.

NSF and the U.S. Department of Energy fund QuarkNet, and the program receives additional support from universities and labs that host QuarkNet centers.

\subsection{Next Generation Science Standards}

Released in July 2011, NGSS describe what all United States students should be familiar with by the time they leave high school. The NGSS framework has three dimensions: core ideas, scientific and engineering practices, and crosscutting concepts such as Energy and Matter. Although released after the first e-Lab was created, it is nevertheless important that the e-Labs align with the framework. Happily, the e-Lab with its platform for students to conduct scientific investigations aligns perfectly with the practices dimension. As NGSS notes, "The practices better explain and extend what is meant by 'inquiry' in science and the range of cognitive, social, and physical practices that it requires." $A$ 
particular e-Lab incorporates various core ideas and crosscutting concepts depending on the studies covered. We have made these ideas and concepts more explicit using NGSS language.

\section{ANATOMY OF AN E-LAB}

Teachers have on-going input into the design of an e-Lab that began with a needs assessment/design workshop attended by 24 teachers in 2004. As recommended, an e-Lab is like a chapter in a book, a teachers' edition, so that a teacher can expect to find the same elements within a similar design"one-stop shopping." e-Labs align with national standards, support guided inquiry and include handson experience. e-Labs offer assessment tools and strategies that align with measurable learner outcomes. The potential exists to build and support professional learning communities.

An e-Lab requires only Internet access and a web browser and contains two main sections, Teacher Pages and Student Pages.

\subsection{Student Pages}

At the heart of an e-lab are stand-alone pages that allow students to reach beyond the classroom to collaborate and publish original work to an audience worldwide. The website provides scaffolding to help students as novice researchers experience an environment of scientific investigation. For example, each e-Lab begins with Cool Science, a short introduction to the experiment. LIGO welcomes students in a particularly appealing video with animations of two black holes orbiting each other in a distant galaxy. The video concludes with an example diagnosing and plotting seismic noise.

With user-friendly navigation and a project map or study guide that supports guided inquiry, the website models scientific research. It provides opportunities to collaborate with other students and access to experts. An e-Lab also accommodates different ability levels and scales of use.

\subsubsection{Getting Started}

When students go to the website, an appealing graphic or animation appears. If students are taking the pre- and post-test, each member of a research group must complete the pre-test before they can start using the website. The tests provide data for teachers and the outside evaluators. Then when students log in, they see the navigation bar and the Project Map.

\subsubsection{Project Map}

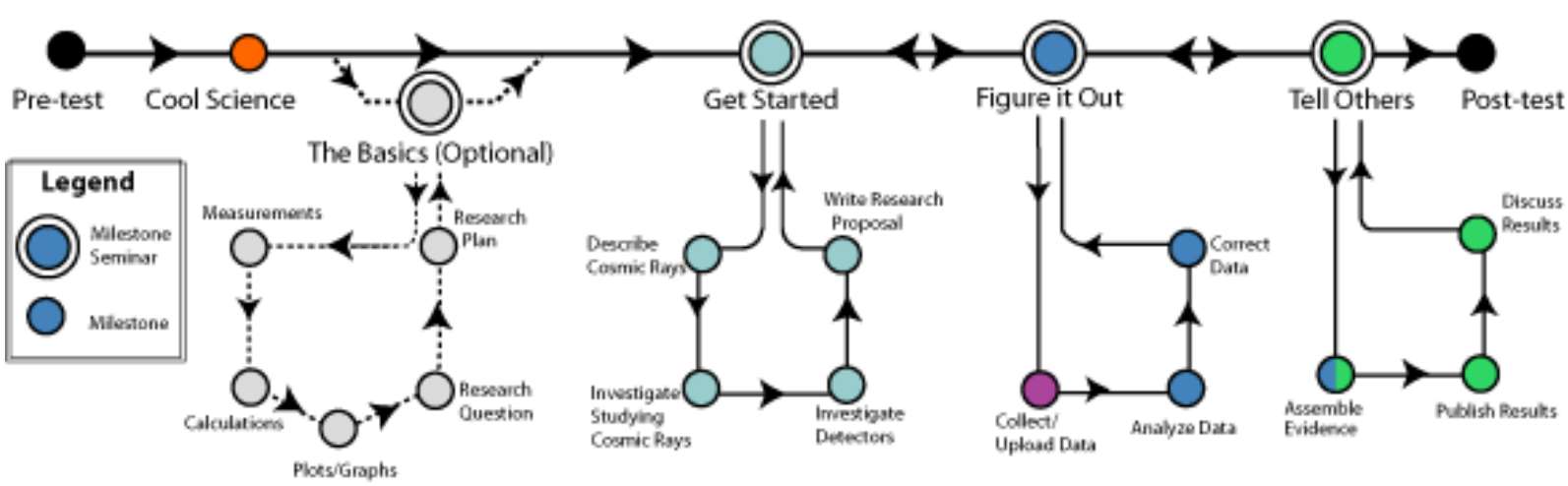

Figure 2. Project Map for Cosmic Ray e-Lab.

The Project Map (Fig. 2) provides a set of milestones that, if followed, will result in a complete investigation. To navigate an e-Lab, student-teams follow the path and complete the milestones. They can hover over each hot spot to preview; click to open. Along the main line students can schedule milestone seminars to meet with their teacher to demonstrate mastery of the material before proceeding to the next branch line.

Project milestones are on the four branch lines. The branch lines as shown on the Cosmic Ray Project Map include:

- The Basics: Reviews basic research skills. 
- Get Started: Prepares the team to design their investigation with links to information about cosmic rays, the detector and a research proposal.

- Figure It Out: Prepares the team to analyze data with links to how to take and upload data, use data search parameters, choose analysis tools, etc.

- Tell Others: Prepares the team to enter into scientific discourse. Links to how to write up the investigation, create a poster and comment on the work of others.

We provide an alternate text version for accessibility.

\subsubsection{Milestones}

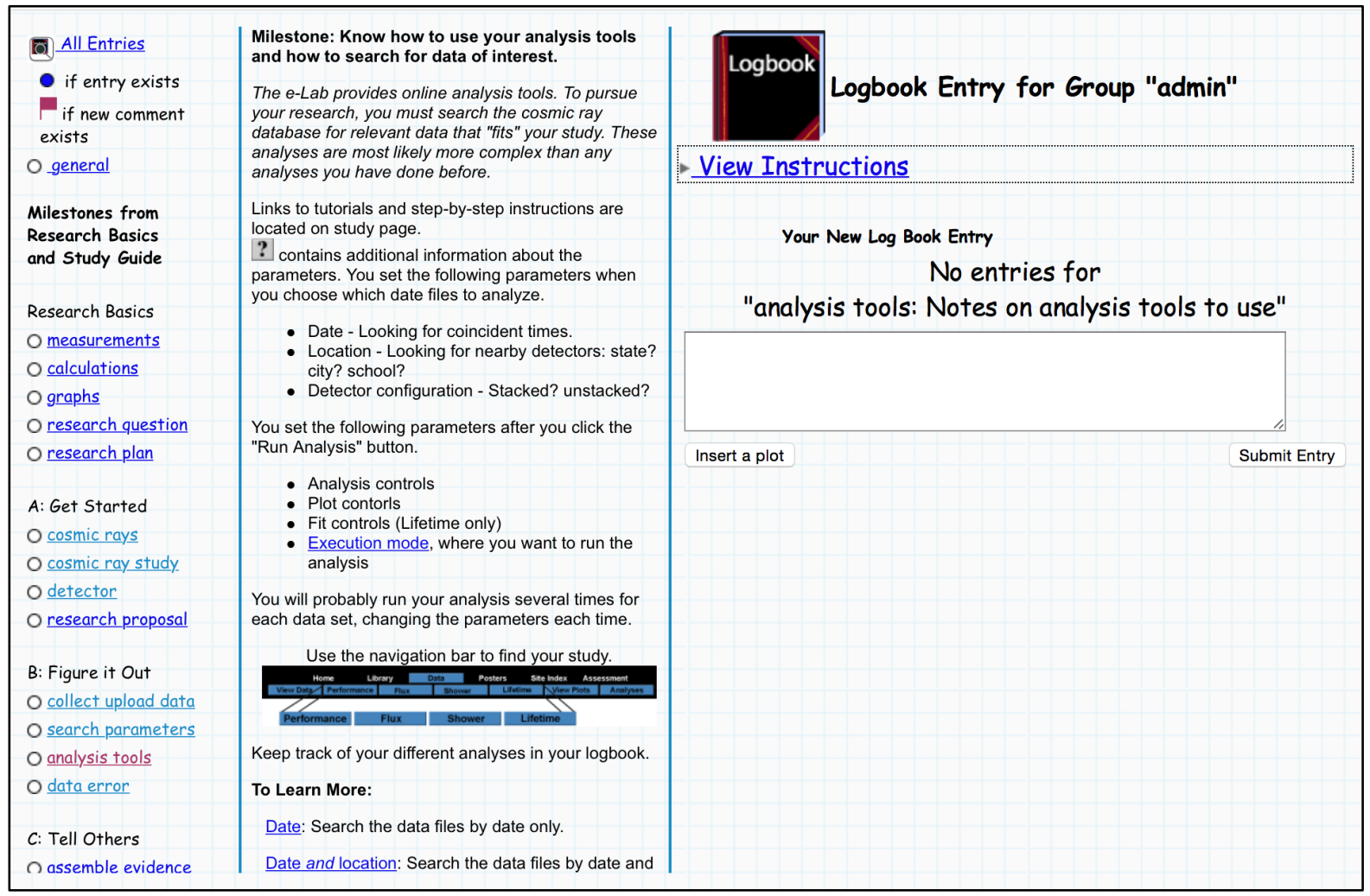

Figure 3. Milestone in Cosmic Ray e-Lab.

Milestones (Fig. 3) allow students to individualize a workflow for their project. As they open a milestone, they can see whether they have already mastered the material and either work through the activities or move along to the next milestone. Each milestone consists of three parts including an index on the left and the student e-Logbook on the right. As teams work through their investigation, they are able to keep their logbook up to date in the same way that a scientist keeps her logbook. The middle section of the milestone contains a short introduction, some discussion, for example, about measurement errors, tips from experts, etc. and links to learn more.

\subsection{4 e-Logbook - Opportunities for Feedback}

Because this is a student-led, teacher-guided investigation, it is important for students to get frequent feedback. As students work through the milestones, they can save their notes in the logbook. They can enter notes for each milestone and into general notes. They can respond to teacher prompts to reflect on their progress. Teachers are able to access this work, track and assess student progress and provide feedback.

Teachers also have a logbook in which they can make notes on their students, teaching strategies, resources, etc. for the next year. To access the logbook, a teacher clicks on the little pencil in the upper right hand corner of the banner.

From their logbook, teachers can access entries:

- By milestone for all their student groups.

- By student research group. 
- What they have written about each research group in their private logbooks.

\subsubsection{Navigation Bar (Fig. 4)}

\begin{tabular}{|l|l|l|l|l|l|}
\hline Project Map & Library & Data & Posters & Site Map & Assessment \\
\hline
\end{tabular}

Figure 4. Student navigation bar for Cosmic Ray e-Lab

Most menu items have submenus (Fig. 5). When a student rolls over the navigation bar, a submenu shows up. This is how it looks when she rolls over "Library" on the Project Map page.

\begin{tabular}{|c|c|c|c|c|c|}
\hline Prolect Map & Library & Data & Posters & Site Map & Assessment \\
\hline Glossary & Resources & Big Picture & FAQs & Site & ps \\
\hline
\end{tabular}

Figure 5. Student navigation bar with submenu for Cosmic Ray e-Lab.

\subsubsection{Data}

The Data tab provides access to the datasets and the analysis tools. (The Cosmic Ray e-Lab has the added feature for a student-team with a classroom detector to upload data.) This is where students do their work. Teams learn about the various studies for a particular dataset and decide on their study. They view and select data for their study, analyze their data, save (and delete) their own plots, and view the plots of others. These plots may give students an idea for their own study.

Students are not limited in the questions they pose as long as their questions can be answered by the data. Students gain insight from manipulating parameters even if the answers are well-known by scientists. They are new to students. Here students find tutorials, screen-casts, step-by-step instructions for using the analysis tools and other assistance. They interpret their data, saving plots for their posters and for other students to view. They also may be able to export their data to use with other analysis tools or to combine with other data.

\subsubsection{Posters}

Students may publish their work as a poster or a paper. We provide a webform for them to enter text, select figures from the website and uploaded images of their own. Associated with posters are areas for comments. By studying posters of other students, they are able to discover, replicate and confirm the work of their peers, much as professional scientists do. This is where real scientific collaboration can begin. Using online tools, students have an opportunity to correspond with other research groups, post comments and questions, prepare summary reports, and in general participate in the part of scientific research that is often left out of classroom experiments.

\subsubsection{Glossary and References}

The website has a glossary and references for the milestones (accessed by clicking on the milestone in the graphical version and on the icon 1 in the text version). Entries show in pop-up windows when a definition or reference is needed. The entire glossary is available from the Library submenu.

\subsubsection{Notifications}

Students can click on the envelopes icon on the upper right hand corner of the banner to see notifications. Teachers can send notifications to students. The e-Lab also notifies the team when data analyses are done. A number in red will indicate the number of notifications available. If it is missing, there are none.

\subsubsection{Resources}

On the Library submenu is a link to references that include:

- Tutorials: Step-by step instructions and tutorials for each of the studies.

- Contacts: Experts and student research groups.

- Online: Other URLs of interest.

- Animations: Aids to help students understand detector, and how the collaboration relies on grid techniques. 


\subsubsection{Site Index and Site Overview}

The student site index has a list in outline format of all the pages on this site available to students.

The student site overview provides a graphical interface to important pages on this site available to students. One can roll over the main sections to see the items available for access in each section. This same interface is accessible from the "Explore!" quick link on the home page. Students may like it as an easy way to get around the website. Here the student has rolled over the Library and exposed

the Library links. (Fig. 6)
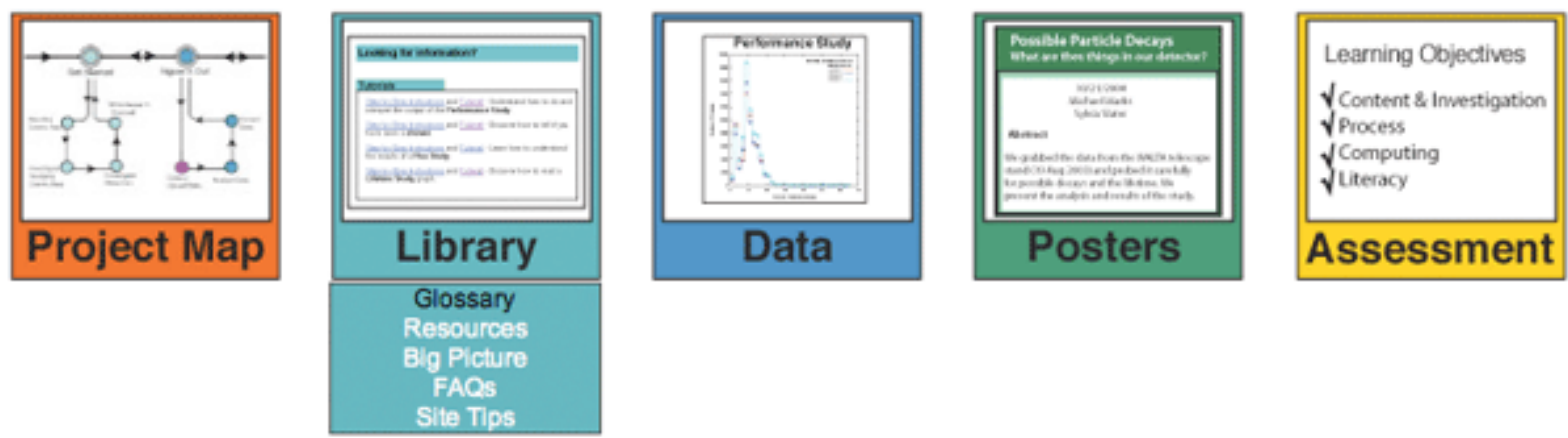

Figure 6. Explore option to navigate Cosmic Ray e-Lab.

\subsection{Teacher Pages}

Teaching tools such as student and teacher logbooks, pre- and post-tests and an assessment rubric aligned with learner outcomes help teachers guide student work. Constraints on interface designs and administrative tools such as registration databases give teachers the "one-stop-shopping" they seek for multiple e-Labs. We provide an extensive stand-alone online area, (fig. 7) or teachers. There are no connections to the teacher pages from the student website.

\begin{tabular}{lccccc}
$\begin{array}{l}\text { Teacher Home } \\
\text { e-Labs Home }\end{array}$ & $\begin{array}{c}\text { Share Ideas } \\
\text { Project Home }\end{array}$ & $\begin{array}{c}\text { Standards } \\
\text { Student Home }\end{array}$ & Site Index & Registration & Publish Posters \\
\hline
\end{tabular}

Figure 7. e-Lab teacher navigation bar

Teacher Home includes:

- e-Lab Summary: Overview of the e-Lab, how it supports student-led, teacher-guided scientific investigations

- Introduction to CMS, cosmic ray research or LIGO: A paragraph or two about the research with links to experiment websites.

- Good Research Questions: Characteristics and examples of good questions correlated with the poster rubric.

- Prior Student Knowledge and Skills: A list includes making measurements and calculations, interpreting graphs, and writing a research question and plan. Refresher references are in the Basics on the student project map.

- Learner Outcomes and Assessment: A list of measurable outcomes. Aligned assessment includes pre- and post-tests, rubrics, e-Logbooks and Milestone Seminars.

- Suggestions for Getting Started: Ways to motivate students to start thinking about a good research question.

- Navigating Students Through the e-Lab: Student website features that help teachers guide student learning while giving students the independence to work at their own pace through their project.

- Help Desk \& Sharing Ideas: Links to help desk and Facebook group.

- e-Lab Technology Requirements: Reminder to check that Javascript and Plug-ins are enabled. Most browsers default to these settings.

Special sections contain registration, a site index, alignment with standards, and a place to publish posters for classroom use. 
While no e-Lab is really complete without a teacher professional development workshop, anyone can access an e-Lab as a guest or request an account to have full access to the site. Only registered users can in turn register their students, preferable in teams of two-three students each. Most teachers need continuing support to use a new instructional website and perhaps adopt new teaching strategies. An important component of that continuing support is a help desk.

\section{CLASSROOM SUCCESS}

Most teachers are comfortable giving lectures and assigning step-by-step labs to be completed during one class period. e-Labs take longer, and when student-teams take the lead, they will not be at the same milestone at the same time. We offer professional development e-Lab workshops to give teachers confidence with the new material, to help them be comfortable to let their students conduct their own investigation and clever to convince school leaders these classes meet standards and school teaching requirements.

Staff teachers or fellows, master teachers, experienced in offering programs for adult learners are instructors in our two or three-day e-Lab workshops. Teachers spend most of the time working through an e-Lab as if they were students. They learn about the dataset and how to use the analysis tools. Many of them experience the discomfort their students may feel planning and conducting their own investigation. As they reflect on this experience, they create an implementation plan for their own classes.

Teachers who have taken an e-Lab workshop tend to implement e-Labs in science clubs, or advanced placement or honors physics classes. Some use them for research projects in a class, or students may design an investigation for a competition. Teachers report that e-Labs do indeed provide students an opportunity to conduct actual research in an authentic way- "gives students a taste of how science actually works."

Michael Fetsko, Miles Godwin High School, Richmond, Virginia, uses the CMS e-Lab as a long-term project in conjunction with a unit on particle physics. He says,

At first, the students are very frustrated by the assignment and find using the website challenging. After a while and a few conversations with me, they realize that the e-Lab is not that complicated and that there are quite a few studies that they can perform. To a certain segment of my students, they are able to find insight into particle physics and particle physics research that they could not experience in any other way. I like the challenge of it and, as a teacher, the fact that I can learn from the work that my students are completing is an added bonus.

Martin Shaffer, Cowley College, Arkansas City, Kansas, uses the Cosmic Ray e-Lab

to do outreach for physics classes at high schools near me. The e-Lab provides a portal to collect, share data and communicate with students while they are conducting research on a variety of questions only young students can ask. The e-Lab provides a tool for me to link my college to local high schools with the intent of inspiring young minds to think about a possible career in physics.

Also, Shaffer uses the e-Lab with his college students in physics and research classes and with students in Math and Science Club.

The national QuarkNet program used posters to evaluate the success students have in their investigations. The latest review showed that $84 \%$ of students completing posters met or exceeded expectations. The most difficult part of an investigation once students determined a good research question was making claims based on evidence and reasoning. Our evaluators reported most problematic for students was not knowing how or not using evidence or only limited data to support claim. They failed to state what figures showed only citing them in their discussion. Almost no one seemed to know how to provide reasoning. Less serious problems included perfunctory or incomplete background information, discussion or conclusion. Students also tended to discuss weaknesses or possible confounding variables without discussing why they could be an issue.

A study of effective teaching practices identified key strategies that lead to successful student investigations as evidenced by their posters. These include:

- Going over all aspects of the e-Lab first or better yet, going through one full example.

- Providing background information through videos and activities (Data Portfolio). 
- Using guided inquiry, checking in frequently rather than presenting the e-Lab and then leaving students to continue on their own.

- Presenting/processing the information as well as science research process; best when teachers have students look at one another's work.

\section{SUMMARY AND PROSPECTS}

e-Labs help students and teachers make sense of data. They are continually under review as new online tools become available, but the scaffolding is available to other experiments. Important considerations are having sufficient data for a variety of investigations and having or creating analysis tools that require only a web browser.

\section{REFERENCES [Arial, 12-point, bold, left alignment]}

[1] Compact Muon Solenoid [Online]. Available http://cms.web.cern.ch

[2] QuarkNet [Online]. Available https://quarknet.i2u2,org

[3] Laser Interferometer Gravitational-wave Observatory [Online]. http://ligo.clatech.edu

[4] Large Hadron Collider [Online]. http://home.cern/topics/large-hadron-collider

[5] Bardeen, M. et al. (2005). "The QuarkNet/Grid Collaboration Learning e-Lab.” Proceedings of the Fifth IEEE International Symposium on Cluster Computing and the Grid, IEEE Computer Society Washington, DC, USA. pp 27-34.

[6] Interactions in Understanding the Universe [Online]. Available http://www.i2u2.org

[7] Next Generation Science Standards [Online]. http://www.nextgenscience.org

[8] Data Portfolio [Online]. https://quarknet.i2u2.org/data-portfolio

[9] International Masterclasses [Online]. http://physicsmasterclasses.org 